\title{
Evaluation of a prototype thermal anemometer for use in low airspeed drying measure calculations
}

\author{
D.P. Martin ${ }^{\mathrm{a}, \mathrm{b}, *}$, J.J. Grant ${ }^{\mathrm{b}}$, J.V. Ringwood ${ }^{\mathrm{a}}$ \\ ${ }^{a}$ Department of Electronic Engineering, National University of Ireland, Maynooth, Co. Kildare, Ireland \\ b Teagasc, Kinsealy Research Centre, Ireland
}

Received 26 June 2001; received in revised form 4 September 2001; accepted 13 November 2001

\begin{abstract}
An indicator of the drying rate of an airflow is the product of airspeed and humidity ratio (or vapour pressure) deficit. Significant sensor errors are present in both the high relative humidity and low airspeed ranges. Also, the margin for error is small relative to the desired drying measure for mushroom growth. The objective of this study was to evaluate the suitability of a low-cost prototype anemometer for use in a mushroom growth environment. A calibrated thermal anemometer was used as a performance reference for the prototype airspeed sensor. Case studies were carried out on two prototype airspeed sensors to determine suitability for use in this drying measure application. The study required the construction of a custom wind tunnel to produce a unidirectional airflow. The sensors (housed in the wind tunnel) were evaluated in an environmental chamber over the range of relevant temperatures. Results show promise for the future application of the prototype sensors subject to the sensors being thermally compensated. Reduction in the cost of a low velocity anemometer by an order of magnitude, would facilitate their purchase and subsequent use for airspeed control by the grower, thus broadening the range of control variables available to the grower. ( $) 2002$ Elsevier Science Ltd. All rights reserved.
\end{abstract}

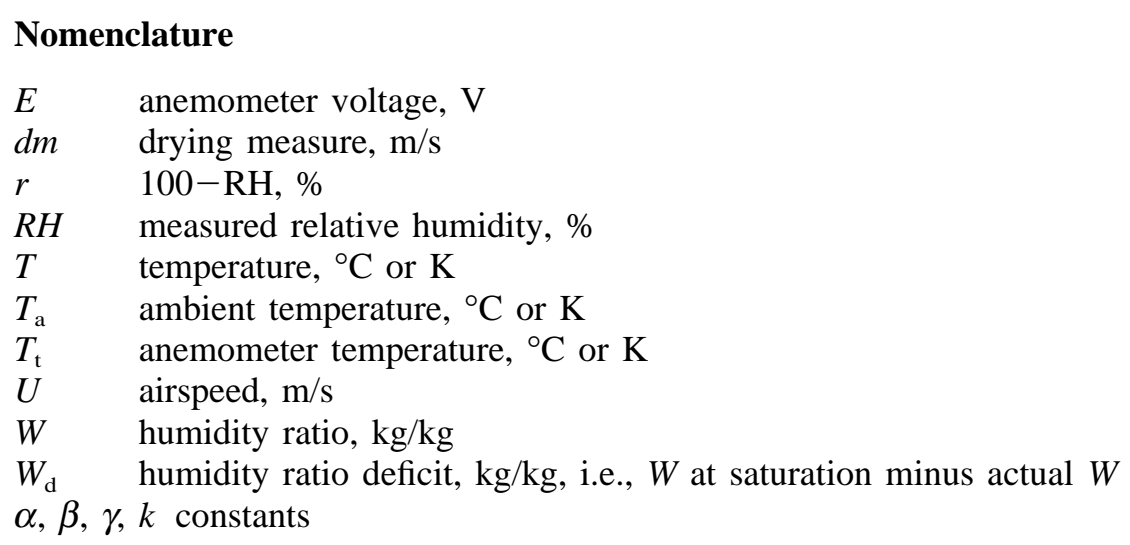

* Corresponding author. Tel.: +353-1-708-6074; fax: +353-1-7086027.

E-mail address: david.martin@eeng.may.ie (D.P. Martin). 


\section{Introduction}

One of the most important means of controlling the development of a mushroom crop is by manipulating evaporative conditions in the growing room. The term 'evaporation power' was used by Bowman [1], and subsequently by Lomax [2], to describe the product of airspeed and vapour pressure deficit, but the use of the term power implies units of Watts, and this could cause confusion, as could the term 'Coefficient of Evaporation' used by Edwards [3]. For the purposes of this study the term drying measure, as defined in Eq. (1), i.e., the product of airspeed and humidity ratio deficit, is used.

$d m=U W_{\mathrm{d}}$

The presence of the airspeed term in the formulation of Eq. (1) underlines the importance of low-velocity airspeed measurement for mushroom growth. It has been found that inappropriate drying can lead to diseased mushrooms. As humidity ratio is indirectly regulated through temperature and relative humidity set-point control, the manipulated variable of choice for drying measure control is airspeed. Hence, airspeed is crucially important to avoid disease during mushroom growth. For a further discussion on mushroom growth, the interested reader is referred to [4]. Note that as a scientific grade anemometer is currently too expensive for the grower, an alternative airspeed measurement solution is necessary. Hence, a low cost thermistor based prototype anemometer was developed in a related project by researchers at University College Galway.

The prime objective of this study was to evaluate the suitability of a low-cost prototype thermal anemometer for use in drying measure calculation during mushroom growth. The anemometer tested was of a thermistor based design for use in low velocity airflow measurement. The prototype thermal anemometer design is very similar to that described in [5].

A crucial element of drying measure calculation for mushroom growth is low velocity anemometry. The measurement of low airspeeds is commonly undertaken using thermal or hot-wire anemometry. Airflow over the crop canopy in mushroom growth is typically less than $0.5 \mathrm{~m} / \mathrm{s}$, which is close to what is often considered as still air.

Thermal anemometer error can increase markedly at low velocities. This is due to the fact that the ratio of convective airflow (due to the heat generated by the hotwire, and specified as $0.03-0.05 \mathrm{~m} / \mathrm{s}$ for the reference anemometer used) to measured airflow increases. Under conditions of a horizontal airflow that was perpendicular to the sensor axis, this is exemplified in Fig. 1.

The growth of mushrooms is undertaken in one of the most protected agricultural environments. Whilst the climate requirements for the growth of a high quality

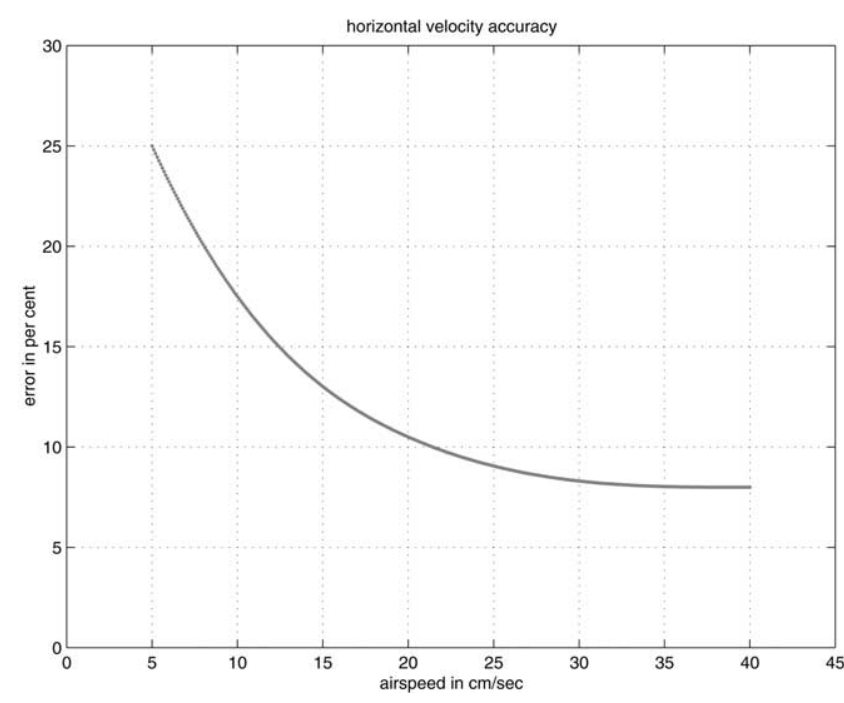

Fig. 1. Specified error for precision thermal anemometer used as reference in this study.

crop are quite well known at a qualitative level, at a quantitative level the knowledge of what constitutes a good drying measure is not well understood. This contrasts starkly with the quantitative knowledge of what constitutes good temperature and carbon dioxide levels. Hence the availability of a low-cost anemometer is very desirable.

The measurement of evaporation, using classical Piché evaporimeters, is not a widespread practice amongst mushroom growers. Measurement of temperature and relative humidity $(\mathrm{RH})$ is quite common, however. Low cost airspeed sensors would be used by growers if they were readily available.

Measurement accuracy using such sensors needs to be evaluated in order to facilitate the comparison of different grower's crop results. The reference used for comparison with the prototypes, was a high-performance thermal anemometer.

Section 2 describes an approximation that simplifies calculation of drying measure in this application and places the relative contribution of each of the relevant sensors errors in context. Following that, Section 3 then briefly recalls that temperature compensation for thermal anemometers is non-linear. The prototypes and the experimental apparatus used to evaluate the prototype anemometers is described in Section 4, and Section 5 presents a summary of the results obtained. A discussion of various aspects of the evaluation and the results is provided in Section 6. Finally, Section 7 then presents the conclusions.

\section{Drying measure calculation}

As the primary objective in mushroom tunnel climate control is thermal regulation, the temperature variable is 
normally taken as being fixed. This facilitates the calculation of the humidity deficit and, in this case, a fixed drying measure is described by a two-dimensional curve.

For any two drying measure curves that show the upper and lower bounds of acceptable drying, the area contained within appears as a banana shape. A typical banana curve with the temperature component fixed is illustrated in Fig. 2. Also shown, using vertical and horizontal bars, is the error contribution due to sensor errors, discussed later in Sections 2.1 and 2.2.

Values of the drying measure that provide good growth of mushrooms are a subject of current research. A drying measure range between 15 and 30 has been used by some commercial growers. The left-hand curve in Fig. 2 illustrates a measure of 15 and the right-hand curve 30 . To demonstrate the potential for drying measure calculation error, typical sensor error contributions are next discussed.

Relatively accurate and low-cost temperature sensors are in widespread use. A sample quoted accuracy of 0.2 ${ }^{\circ} \mathrm{C}$ in the range $0-70{ }^{\circ} \mathrm{C}$ being quite typical. The temperature sensor error in effect provides an offset to the banana curve, i.e., shifting the curve by a small amount. Such a shift is very small, as shown in Fig. 2 by the overlapping centre-points of the triangle markers, with the unshifted curves. Therefore this type of sensor error may be ignored.

\subsection{Humidity sensor error contribution}

To account for the temperature dependency of water vapour in air, vapour pressure or humidity ratio deficit is often used for calculation purposes. Note that the secondary climate control objective in a mushroom tunnel is humidity control. If it is assumed that the primary climate control objective in a mushroom tunnel, i.e., temperature regulation, is readily achievable, then a dry-

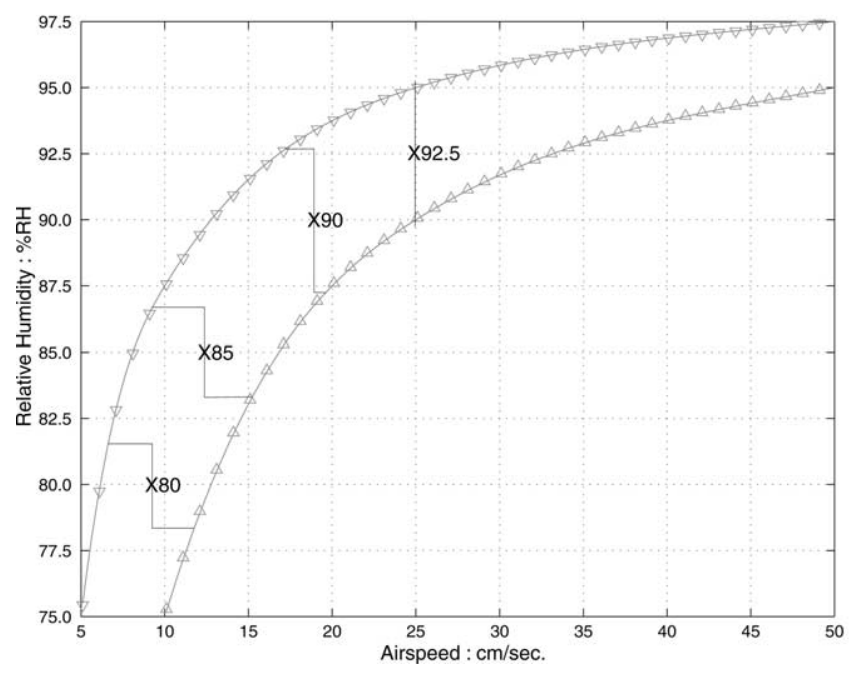

Fig. 2. Sample banana curve $\left(17^{\circ} \mathrm{C}\right)$. ing measure calculation simplification is available. The moisture component of drying measure, humidity ratio deficit, can be approximated by a relative humidity (RH) deficit in the limited temperature range where humidity is controlled, as per Eq. (2). Thus leading to a drying measure reformulation, for a constant temperature as per Eq. (3).

$r=100-R H$

$d m=U \mathrm{r} ; T=k$

A commonly used humidity sensor in mushroom tunnels is of a capacitance based polymer design, due to its low cost and maintenance requirements. The variation in the accuracy of these sensors is significant both between different manufacturers and between different parts of the measurement scale. Typically, sensor error is greatest at high $\mathrm{RH}$, which is the region of interest in this application.

To highlight the maximum allowable airspeed error component, representative drying measure points are illustrated in Fig. 2 with an ' $\mathrm{X}$ '. A vertical error band for relative humidity (RH) error and horizontal error band for airspeed error is also illustrated. The RH error band uses 3\% accuracy at 90\% RH and above, and 2\% accuracy below $90 \% \mathrm{RH}$. These figures are typical of a well specified sensor's accuracy in the high RH domain. However, note that practical laboratory testing of similar types of sensor showed that manufacturer's quoted accuracy was better than that found in testing by a factor of approximately three [6]. Hence it is recommended to treat quoted accuracy figures for this type of humidity sensor with a degree of caution.

\subsection{Sensor accuracy trade-off}

The temperature sensor error contribution is significantly less than that of typical RH sensors and far less than that of a precision airspeed instrument, so it is assumed that the effect of temperature sensor errors as a contributing factor to drying measure calculation error may be neglected. What then is the effect of the $\mathrm{RH}$ sensor error as a contributing component to the total error in drying measure calculation?

In Fig. 2, consider the RH set-points marked with an ' $\mathrm{X}$ ' mid-way between the extremes of the banana curve. Using an error margin of 2 or 3\%, respectively for below and above a $90 \% \mathrm{RH}$ value, the possible $\mathrm{RH}$ value range for the set-points listed is indicated by a vertical line. Now that the known RH sensor errors have been clearly identified, consider the corresponding airspeed error (indicated by a horizontal line) that maintains the desired drying measure set point (the ' $\mathrm{X}$ ') within the extremes of the banana curve.

Starting at the $80 \%$ set-point, i.e., 'X80' from Fig. 2, 
Table 1 lists the available error span to maintain the setpoint drying measure within the extremes of the banana curve.

Consider the $92.5 \%$ set-point in Fig. 2 and note that either the positive or negative maximum $\mathrm{RH}$ sensor errors are by themselves sufficient to move the drying measure point beyond the border of the banana curve. This threshold level sharply illustrates the difficulty above the 'knee' point of the banana curve.

So, for a fixed temperature, the humidity/airspeed sensor error contribution conflict can be summarised as follows.

As air humidity decreases,

- humidity sensor error decreases, but, to maintain a constant drying measure,

- airspeed has to correspondingly decrease.

As airspeed decreases,

- the airspeed sensor error increases.

Hence a trade-off exists between lower humidity sensor error and higher airspeed sensor error for a fixed drying measure. A corresponding conflict is observed if we view the problem in airspeed terms.

A very small proportion of growers currently practice a drying measure strategy of high humidity in combination with high airspeed (an energy intensive approach). A simple recommendation to use lower humidity and airspeed levels is insufficient to meet every grower's requirements.

\section{Thermal anemometer compensation}

Various velocity calibration equations have been developed for hot-wire anemometers. King's law [7] expressed in Eq. (4) is the recognised equation applying to a hot wire anemometer in the case of an isothermal, unidirectional flow and is often used as a calibration relationship for thermal anemometers, (with $\gamma=0.45$ or $0.5)$.

$E^{2}=\alpha+\beta U^{\gamma}$

Table 1

Available airspeed error: RH sensor error in the 15-30 range

\begin{tabular}{ll}
\hline RH set-point (RH error) & Airspeed error span available \\
\hline $80 \%( \pm 1.6 \%)$ & $54.2 \%$ \\
$85 \%( \pm 1.7 \%)$ & $47.6 \%$ \\
$90 \%( \pm 2.7 \%)$ & $13.3 \%$ \\
$92.5 \%( \pm 2.775 \%)$ & 0 \\
\hline
\end{tabular}

Compensation of the variation of thermal anemometer velocity output with temperature has been done with correction factors [8] using equations such as those proposed by [9]. Eq. (5) [10] is one example of this form.

$E^{2}=\left(\alpha+\beta U^{\gamma}\right)\left(T_{\mathrm{t}}-T_{\mathrm{a}}\right)$

The velocity calibration form expressed in Eq. (6), is for isothermal forced convective flow. This suggests itself for use in a wind tunnel in an environmental chamber, which was the experimental configuration used in this study.

$U=\left[\left(E^{2}-\alpha\right) / \beta\right]^{1 / \gamma}$

Determining the constants in Eq. (6) has been solved by a Taylor series expansion method [11] and values of $\gamma$ found using this method relate $U$ to $E$ up to a power of 5.7. Alternative forms of calibration relations have been proposed and compared [12,7], including King's law, i.e., Eq. (4). The comparison of [12] is based on airspeed data greater than $1 \mathrm{~m} / \mathrm{s}$, and hence does not include the low airspeed range of interest for this study. Hence the results are not directly related. Note the nonlinear form of all the equations proposed.

\section{Prototypes and experimental apparatus}

The prototype anemometers evaluated were developed by different researchers in a previous study and provided for the study by a commercial developer who was considering them for field use. To simulate low-cost production methods, they were provided in an uncalibrated state. Examination of the circuit diagram provided, showed that the thermistor's output was connected to a programmable integrated circuit. A bridge circuit using a second thermistor for thermal compensation was evidently not being used. The thermistor was connected to the circuit by flying leads and potted in a readily available, stainless steel prefabricated housing. The potting compound was poured such that the thermistor was exposed to the airflow, as shown in Fig. 3. The design of the prefabricated housing requires that the flow direction is known.

The sensor head of the prototype anemometers supplied was sensitive to the airflow direction, in both pitch and yaw. This is a result of the fact there is one yaw and pitch airflow axis (path) through the sensor head and over the thermistor that has minimum resistance. Airflow resistance increases as the yaw or pitch of the sensor varies from 0 to $90^{\circ}$ away from the airflow being measured.

Therefore, a critical requirement in sensor head use was to ensure that the measured airflow was perpendicu- 




Fig. 3. Sensor head design for prototype and reference anemometers.

lar to the sensor head (in both pitch and yaw). This requirement does not facilitate practical use in this form. The sensor head of the reference thermal anemometer has a less demanding requirement, due to its spherical construction, and open cage guard design. The two types of sensor guard are illustrated in Fig. 3 .

Preliminary experiments using the ventilation system in a standard length mushroom tunnel proved inadequate as a means of providing a controlled environment both in terms of low airspeed and temperature. The airspeed control mechanism in the tunnel was a boost duct supply with individual fan speed control on each duct [13]. The temperature control mechanism is a thermostatically controlled heater and cooler. This approach has a known problem in achieving temperature control in a flowing stream [14]. It is known that a non-isothermal jet's airspeed is turbulent in nature and hence difficult to control.

A further difficulty arose due to the omni-directional airflow response of the reference anemometer in comparison to the directional response of the prototype. Sample data from this experimental set-up is illustrated in Fig. 4. Note the distinct 'flare' of data that does not follow the linear trend of the bulk of the data.

It is assumed that the data in the flare shows a sensor response that doesn't increase during periods where there is an off-axis increasing airflow, due to the shielding effect of the sensor guard. However, the omni-directional reference anemometer does respond to the off-axis airflow, thus introducing non-linear data into the initial experimental results.

To control the directional element of the airflow across the sensor heads, a decision was made to use a wind tunnel and place it (with the sensors) in an environmental chamber. This approach separated the experimental control problem into two distinct parts: control of airspeed and direction, and control of temperature. Alternatives, e.g., temperature controlled wind tunnels such as that used by [15], were unavailable for use in this study.

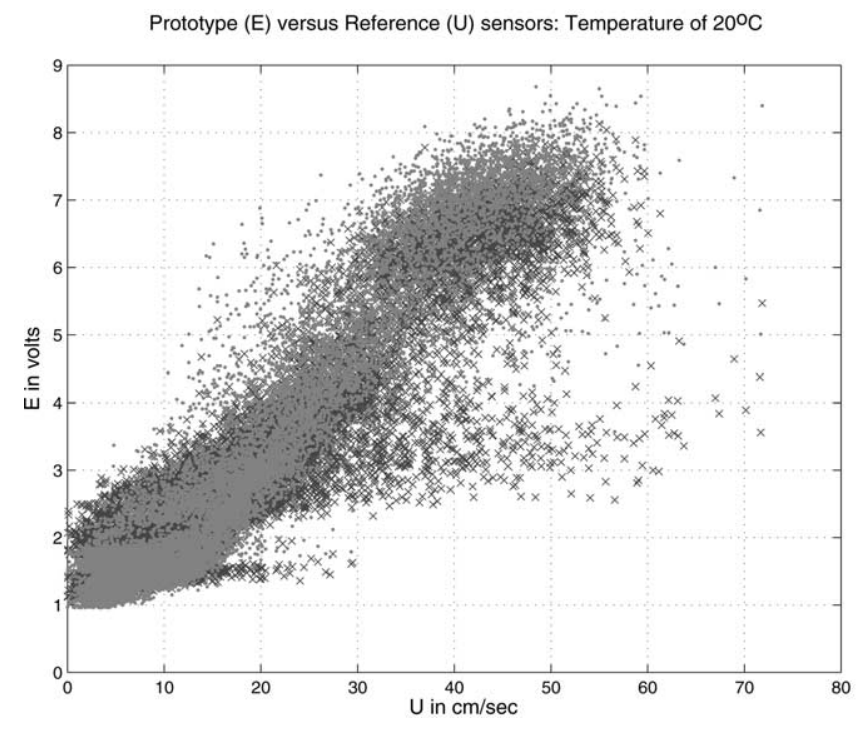

Fig. 4. Omni-directional data for prototype 1(X) and prototype 2(.), at $20.2{ }^{\circ} \mathrm{C}$.

\subsection{Airflow control—wind tunnel}

Although small-scale commercial wind tunnels were available, a space constraint (approx. $1.5 \mathrm{~m}$ maximum length) from the environmental chamber, prevented their use. Furthermore a common design feature of the commercially available wind tunnels was a jet discharge into open space. Due to the low velocity (and consequent momentum) of the airflow in this study, this design approach cannot guarantee the airflow directionality desired for evaluation.

Therefore, following the example of [16] a custombuilt modular anemometric calibration wind tunnel was fabricated that could enclose the sensors in a controlled airflow. The disassembled wind tunnel is shown in Fig. 5.

The objective of the custom wind tunnel design was to provide a reasonably steady unidirectional airflow for the thermal anemometer sensors over the range of air- 


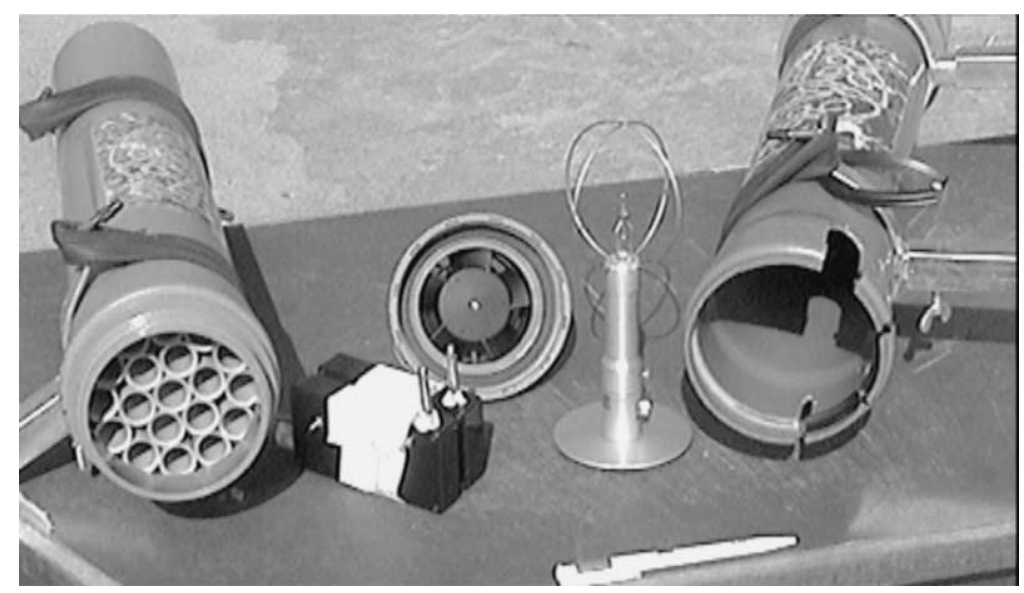

Fig. 5. Wind tunnel and anemometers.

speeds in use for drying measure control in mushroom growth.

To minimise environmental chamber internal wall effects on the local wind tunnel airflow, a clearance of one metre was maintained all round the wind tunnel in the environmental chamber. Whilst it is generally desirable to incorporate a vena contracta in a wind tunnel, the longitudinal space constraint meant that one could not be incorporated in this design. This omission, follows the practice of several studies that have used laminar flow in a circular pipe as a calibration tool for low velocities. A discussion of laminar pipe-flow calibration can be found in [7]. Hence the omission of a vena contracta in this design is not significant.

The wind tunnel was constructed of the following components. Its layout is illustrated in Fig. 6.

- Optional intake air filter-can be used as flow restrictor if required (not used in this study)

- Inlet air straightener
- Co-planar mounting of the reference anemometer sensor head, two prototype anemometer sensor heads, and a temperature probe, between the two sections of the wind tunnel (illustrated in upper left of Fig. 6)

- Main body that can be split in half (illustrated in upper right of Fig. 6)

- Exit air straightener

- Exhaust fan

- Wind tunnel supports

Both the inlet and exhaust air straighteners were constructed of a honeycomb of 19 PVC $19 \mathrm{~mm}$ tubes that were glued together as a snug fit in either section of the wind tunnel. The tubes were arranged as concentric circumferential rings of 1,6 and 12, as recommended in standards [17]. Each tube had a bevelled inlet and outlet and was of length approximately two times the wind tunnel diameter $(110 \mathrm{~mm})$.

Both sections of the main body of the wind tunnel were constructed from standard $110 \mathrm{~mm}$ PVC ducting.

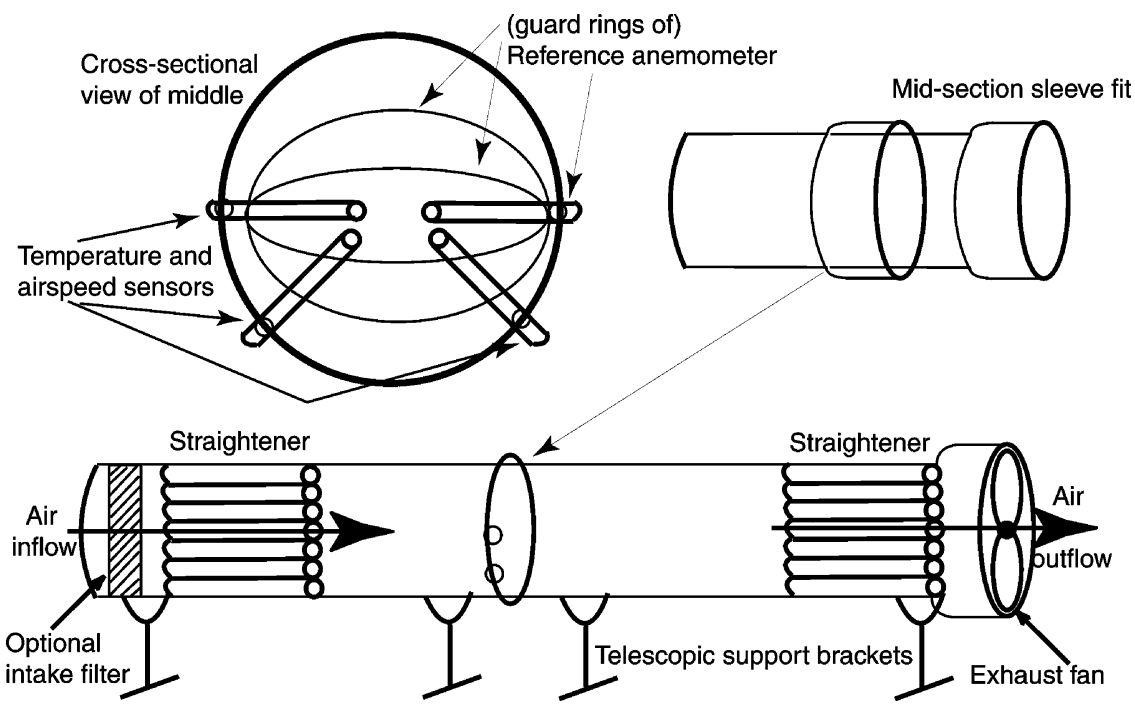

Fig. 6. Wind tunnel layout. 
The ducting comes supplied in standard lengths with a wider (relative to the main body) diameter sleeve (female end) to facilitate duct jointing. Two lengths of ducting cut to approx. $0.75 \mathrm{~m}$ were used as the main sections in the body of the wind tunnel.

Due to the overall length constraint, some section length compromises had to be made, compared to those recommended in the standard, e.g., the length of the air straightener was half the recommended length. However, the main body section lengths did meet the recommended lengths.

The exhaust fan used was a $12 \mathrm{~V} \mathrm{dc}, 80 \mathrm{~mm}$ diameter, $8.8 \mathrm{l} / \mathrm{s}$ axial flow fan. A standard fan finger-guard was attached to the exposed side of the fan to prevent possible injury. The fan was mounted on a machined flange that was fitted to the end of the main body of the wind tunnel. The fan was used in exhaust mode to minimise turbulence in the wind tunnel. This is a feature used in other low velocity wind tunnel designs [18].

Four (two for each part of the wind tunnel) standard $110 \mathrm{~mm}$ duct telescopic ' $\mathrm{Y}$ ' mounts were used as the wind tunnel supports. This allows for the adjustment of the height of the wind tunnel above its mounting surface, a table in the environmental chamber. Both internal, to minimise surface disturbance to the airflow, and external, in preparation for sealing, cleaning with alcohol swipes was undertaken prior to assembly.

Air leakage prevention was approached via several modes during the assembly operation. The first mode of sealing, for the non-permanent junction of both sections of the main body, consisted of two operations. The first sealing operation was between either section of the wind tunnel body and relied on an in situ glued internal rubber compression joint (the standard used for such a duct junction). The second operation was external sealing using non-setting putty.

The second mode of sealing was between the four sensor heads and the wind tunnel. For each sensor head a hole or slot was machined in the wider diameter, female part of the wind tunnel. A corresponding slot was also machined in the male part of the wind tunnel for each sensor head. All the holes and slots were precision machined to ensure a close compression fitting. Prior to both parts of the wind tunnel being mated together the sensor heads were secured in position, as close together, in the same plane, to the centre of the wind tunnel without touching, by the external application of non-setting putty. Once the two parts were mated together, the midsection joint was externally sealed using non-setting putty. The final mode of sealing for all other permanent joints, e.g., fan to flange, flange to wind tunnel, etc., was external sealing using hard-setting silicone sealant.

When construction of the wind tunnel was complete, the flow field in the measurement mid-section was examined. A smaller anemometer of lower accuracy was used to increase the sample resolution in the flow field.
Although its output was linearly compensated using the manufacturer's data for the airspeed range found in the flow field examination it was found to have a bias, e.g., $0.4 \mathrm{~m} / \mathrm{s}$ reading for a $0.35 \mathrm{~m} / \mathrm{s}$ reading on the reference. Averaged measurements ( $30 \mathrm{~s})$ at seven positions on four diameters were used to examine the flow field.

It would have been preferred to select the number and position of measuring points used for sampling the flow field following the requirements of standards [17] (using the log-Tchebycheff method). Practical limitations restricted adherence to the standard's requirements, due to the relatively small size of the wind tunnel, e.g., internal diameter of approximately $105 \mathrm{~mm}$, as compared to the greater than or equal to $300 \mathrm{~mm}$ data discussed in [17]. Because of the sample length of the precision reference anemometer, i.e., approx. $35 \mathrm{~mm}$, only three flow field samples could be taken across any single diameter of the tunnel with the reference anemometer. This would not meet with the standard's requirement for a minimum number of five samples per radius, or eleven per diameter. This was why the lower accuracy anemometer (albeit with a smaller sampling length) was used. The size of this sensor still limited the number of samples to seven. These positions were equally spread across the diameter at approx. $15 \mathrm{~mm}$ intervals, starting $7.5 \mathrm{~mm}$ from the inside wall.

It was presumed that the dominant flow field influence to the flow in the mid-section would be the mid-section's nearest neighbours, i.e., the two air straighteners shown in Fig. 6. Relative rotation of the exit straightener to the measurement plane confirmed that this had some effect. Therefore, the angular sampling interval was chosen so that the flow field in the mid-section was sampled (within the limits of the diametrical resolution discussed above) such that there was both a fully obscured (by one straightener), and fully unobscured view of the intake from the mid-section plane of view. It was felt that this should correspond to any minimum or maximum flow condition, respectively. For the straightener's outside ring of twelve tubes, shown in the left part of Fig. 5, this condition is satisfied by a sampling angle interval of $45^{\circ}$, which results in alternation of minima and maxima for successive sample diameters. A sampling angle interval of $90^{\circ}$, gives the same result for the straightener's inner ring of six tubes. As the higher frequency sampling interval $\left(45^{\circ}\right)$ is an integer multiple of the lower one $\left(90^{\circ}\right)$, it was used as the angular sampling interval. Note that because the near radial sample of one diameter's samples, e.g. $\left(0^{\circ}\right)$, corresponds to the far radial sample of that sample angle plus $\pi\left(180^{\circ}\right)$, only sample angles in the range $0-180^{\circ}$ are required to cover the complete mid-section plane.

For a fixed nominal airspeed of $0.35 \mathrm{~m} / \mathrm{s}$, the maximum and minimum absolute difference (and standard deviation) from the mean of any one diameter's measurements, excluding the two wall effect, end 
measurements, were $87.7(28.2) \mathrm{mm} / \mathrm{s}$ at $45^{\circ}\left(45^{\circ}\right)$, and $63.8(19.9) \mathrm{mm} / \mathrm{s}$ at $135^{\circ}\left(90^{\circ}\right)$, respectively. The overall maximum absolute difference (and standard deviation) from the mean of all diameters' measurements, excluding all the wall effect, end measurements, was 89.2 (23.3) $\mathrm{mm} / \mathrm{s}$. Much smaller differences were found at the nominally equi-radial positions where the test and reference anemometers were located during the evaluation of the prototypes. However, the physical size resolution restriction on the anemometer head prevented this being accurately quantified.

\subsection{Environmental chamber and instrumentation}

The largest environmental chamber available for use during the study, had approximate dimensions of $3.56 \times 2.38 \times 2.62 \mathrm{~m}$. The temperature slew rate response of the environmental chamber was specified to be in the order of $1-2^{\circ} \mathrm{C} / \mathrm{min}$. To reach the humidity set-point a time response in the order of 30-40 min was specified.

Both temperature and humidity instrumentation was available within the environmental chamber, but since, higher accuracy, calibrated, stand-alone instruments were available, these were used instead. This approach had the advantage that local measurements could be made with respect to the wind tunnel, rather than relying on even spatial environmental conditions within the chamber.

Temperature measurement was made using a combined meter/logger. The temperature probes used provide a resolution of $0.05{ }^{\circ} \mathrm{C}$, which is well within the $0.2{ }^{\circ} \mathrm{C}$ required in [19]. The logger recorded all the data measured in the experiment at a sample interval of one second. In addition to a date and time stamp, the logged data consisted of readings from the following sources.

Wind tunnel sensor plane

- two prototype anemometer sensors

- reference anemometer sensor

- sensor plane air temperature probe

Wind tunnel air straightener inlet

- inlet air temperature probe

- inlet air humidity probe

A commercially available, temperature compensated, calibrated anemometer was used as the reference airspeed sensor. A four-wire protective guard ring protects the sense element in this sensor. The airspeed and sensor air temperature sense elements were placed perpendicular to the airflow, at the same radial elevation, in the same plane, within the reference guard ring.

A combined RH and temperature sensor was used to monitor inlet air $\mathrm{RH}$ and temperature. The temperature resolution of this meter was $0.1{ }^{\circ} \mathrm{C}$, so a second (higher resolution) temperature probe was used to monitor the inlet air temperature. The $\mathrm{RH}$ resolution was $0.1 \% \mathrm{RH}$, with a dew-point accuracy of $0.2{ }^{\circ} \mathrm{C}$.

An adjustable laboratory low voltage DC power supply was used to power the exhaust fan and provide fan speed control.

\subsection{Experimental procedure}

The experimental procedure followed met with the 17point checklist provided by [20]. Additional points of interest are described as follows.

The environmental chamber had a table upon which the wind tunnel was placed. The wind tunnel's position was set to provide $1 \mathrm{~m}$ clearance all round, with respect to the entry and exit planes.

The sensors were positioned in their respective locations. The cable leads for the fan power supply and all of the sensors being recorded were laid out and fixed in position. The cables were supplied through the cable access port provided in the environmental chamber, and connected to the appropriate instrument in the environmental laboratory.

The recording and chamber temperature and humidity readings were cross-checked. Ten isothermal and two isoflow experiments were then undertaken.

\section{Results}

\subsection{Isothermal experiments}

Ten isothermal experiments were made at $1{ }^{\circ} \mathrm{C}$ intervals and spanned the temperature range of interest used in mushroom growth $\left(16-22{ }^{\circ} \mathrm{C}\right)$ with a clearance band of $1.5^{\circ} \mathrm{C}$ above and below this range, i.e., 14.5, 15.5, ..., $23.5^{\circ} \mathrm{C}$.

For each isothermal experiment the airflow was set at a nominal $0.6 \mathrm{~m} / \mathrm{s}$ (as read by the reference anemometer) and data logged for approximately two minutes. The nominal airflow was then decreased by $0.1 \mathrm{~m} / \mathrm{s}$ and further data (again of two minutes duration) logged. These steps were repeated down to a nominal airflow of $0.1 \mathrm{~m} / \mathrm{s}$.

From the $0.1 \mathrm{~m} / \mathrm{s}$ airflow level, the above steps were repeated, but this time the airflow was increased in steps of $0.1 \mathrm{~m} / \mathrm{s}$ back up to $0.6 \mathrm{~m} / \mathrm{s}$. A sample plot of the data thus gained is illustrated in Fig. 7, for a temperature of $23.5^{\circ} \mathrm{C}$. This approach was employed in an attempt to detect any hysteresis present in the sensors' response.

Note that as the airflow through the wind tunnel decreases, there is less cooling of the thermal anemometers. Hence the air temperature at the sensors increases as airflow decreases and vice versa. 


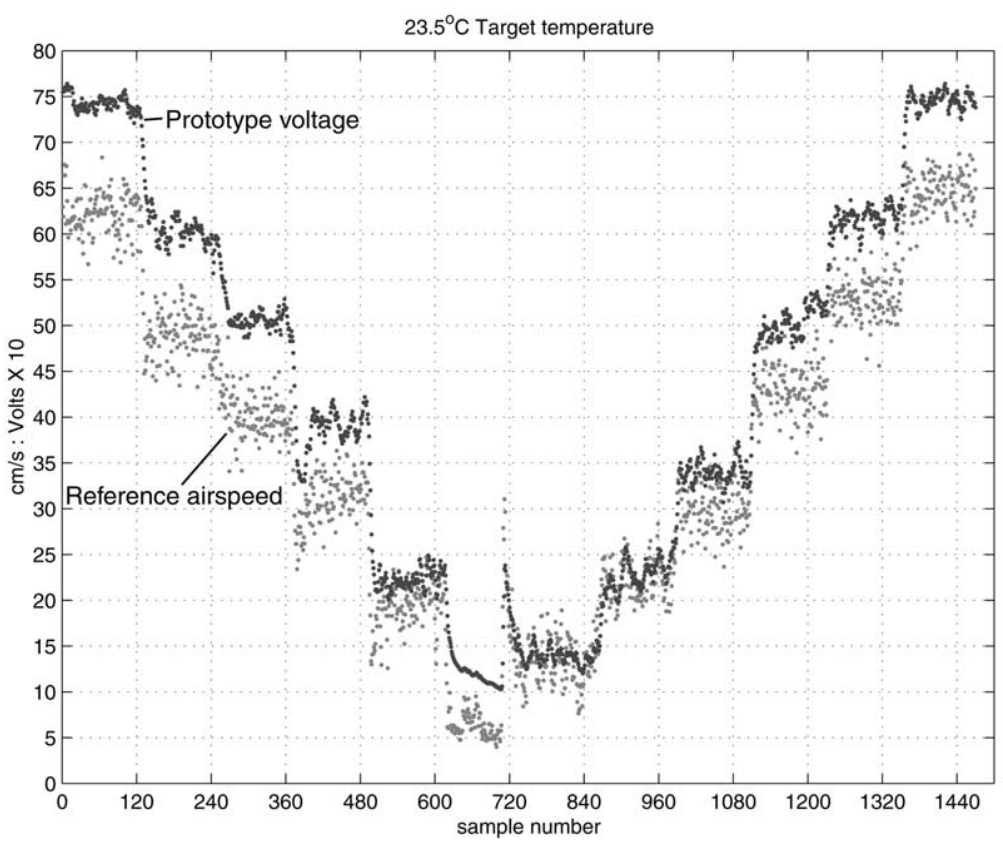

Fig. 7. Sample reference and prototype outputs $\left(23.5^{\circ} \mathrm{C}\right.$ isothermal data).

\subsection{Isoflow experiments}

Due to the limited time availability of the environmental chamber, only two isoflow experiments were made at a nominal airflow of 0.15 and $0.3 \mathrm{~m} / \mathrm{s}$. These values were selected as being typical of those used in practice.

In each isoflow experiment the airflow was first set to the nominal value, as read by the reference anemometer. Then the air temperature passing over the sensors was decreased from $23.5{ }^{\circ} \mathrm{C}$ down to $14.5{ }^{\circ} \mathrm{C}$, and then increased back up to $23.5^{\circ} \mathrm{C}$. A sample plot of the data thus collected is shown in Fig. 8.

Note the clear change in prototype output in Fig. 8,

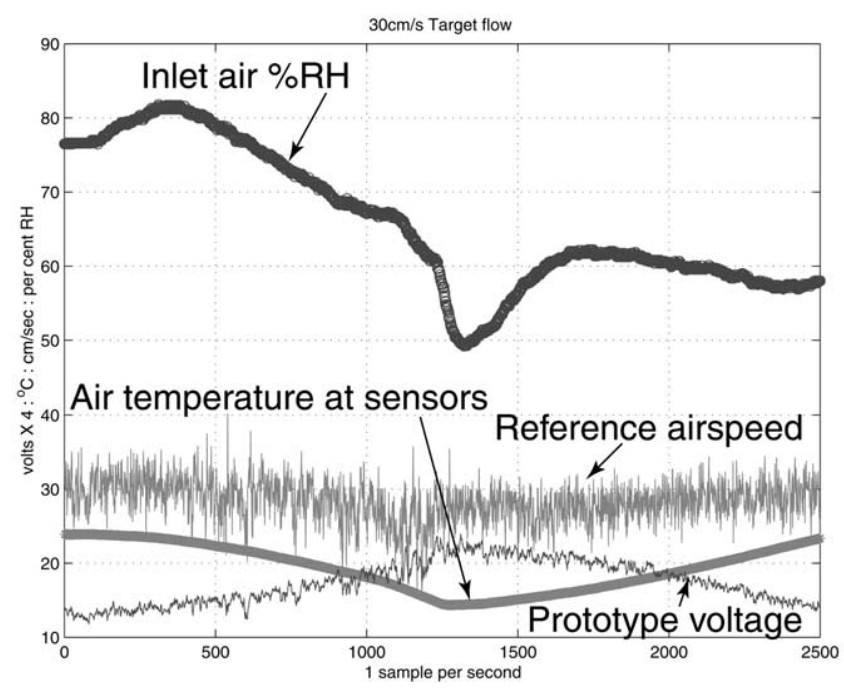

Fig. 8. Sample isoflow data $(0.3 \mathrm{~m} / \mathrm{s})$. as temperature is decreased and then increased. Also, note that no correlation between relative humidity and prototype output was found in the study.

The thermal slew rate at the airflow sensors for this part of the experiment was manually controlled to be approx. $0.5^{\circ} \mathrm{C} / \mathrm{min}$. This rate was used, as no faster crop level thermal slew rate had been observed in measurements made during mushroom growth.

\subsection{Data collection and processing}

The data from each sensor type, temperature, humidity and airspeed, used in the experimental apparatus was recorded at a $1 \mathrm{~s}$ sample rate. The zero point offset $(0.25$ V) for the two prototype airspeed sensors was first subtracted from their respective outputs. An offset is a characteristic of hot-wire anemometers [21].

It was known that the reference anemometer sensor had a faster response to a change in airflow (for $0.1 \mathrm{~Hz}$, the $90 \%$ time constant specified was $0.8 \mathrm{~s}$ ) than that of the prototypes (typical time constant specified was approx. $15 \mathrm{~s}$ ). This resulted in the reference anemometer passing turbulent airflow changes with greater amplitude. Hence the reference airspeed data appears to be more turbulent.

Preliminary evaluation of the experimental data, for tracking performance, was undertaken using both raw and averaged data. Fig. 9 illustrates prototype and reference data averaged, using the raw data shown in Fig. 7. There are several sources that suggest the use of time averaging in this application. The airflow under examination is turbulent and the corresponding measurement data is non-stationary. For a standard Robinson cup or 


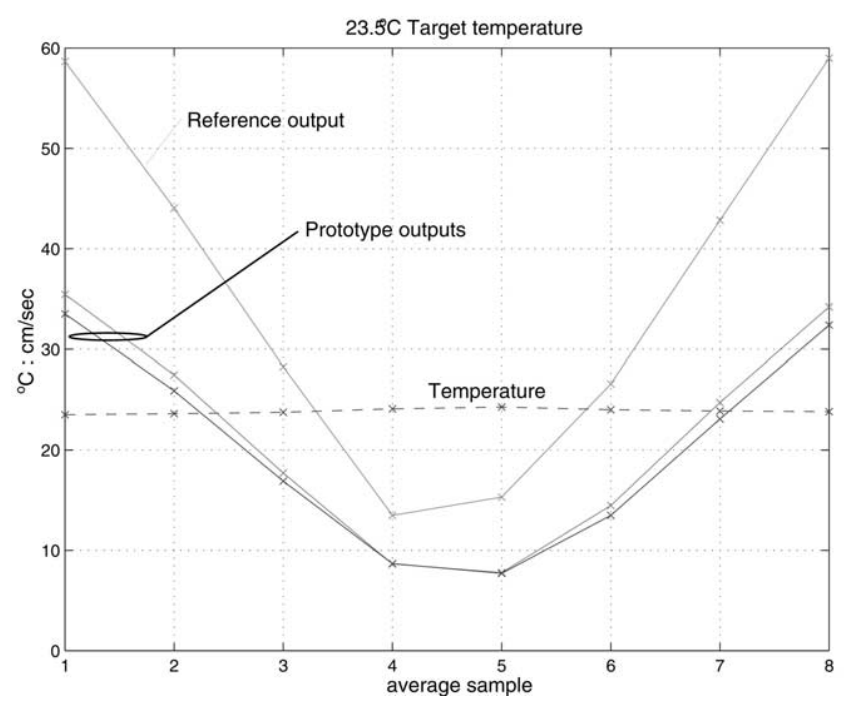

Fig. 9. Sample isothermal data averaged for $180 \mathrm{~s}$.

vane type anemometer, the US weather service averages a minute's data [22]. It's known that the selection of sampling frequency and duration is difficult [23], and a corresponding standard, to the US one, or recommendation for hot-wire anemometers is unknown to the authors. Whilst a required upper frequency of $1 \mathrm{~Hz}$ for low velocity anemometers has been proposed [21], a time of three minutes is suggested for measuring mean velocity. This contrasts with time-mean averages of 10 $30 \mathrm{~s}$ which are recommended for use in hot-wire calibration [7]. Yet another proposal [24] is an integration time of 15-20 min. Note that this proposal refers to a study undertaken in a ventilated room where turbulence intensity was about $30-40 \%$.

An analysis [25] of the US averaging recommendation indicates that significant error accompanies its use. Therefore the question of what is an appropriate time averaging span arose.

It was decided to have corresponding time constants for drying measure calculation, i.e., the matching of the anemometer sensor time constant of that of a protected RH sensor (e.g., $180 \mathrm{~s}$ ). Hence the recommendation of [21] was adopted and is considered more than adequate as the use of three minute averaging has given repeatable results [26] for many years in practice.

\section{Discussion}

\subsection{Calibration}

It is known that both the velocity calibration and temperature calibration of a hot-wire anemometer are nonlinear [27], and that standard calibration procedures, i.e., Pitot tube, are inaccurate for the small signals, less than $1 \mathrm{~m} / \mathrm{s}$, that are characteristic of this application. The ref- erence sensor was calibrated in a low velocity wind tunnel using a laser Doppler anemometer as reference, with an estimated accuracy of $1 \%$. This is claimed to be the highest accuracy anemometric source that is accessible [28]. A study of accuracy of measurements made using this (and other) thermal anemometer(s) was undertaken by [15]. The output of the reference anemometer follows the linear relationship of Eq. (3) with an accuracy of $0.01 \mathrm{~m} / \mathrm{s}$ or $5 \%$ of reading in the range $0.05-1 \mathrm{~m} / \mathrm{s}$.

It is important to note that all hot-wire anemometers induce a natural convective flow due to self-heating [18]. It's claimed that for the reference anemometer no errors from this phenomenon occur above an air velocity of $0.05 \mathrm{~m} / \mathrm{s}$ in a horizontal flow (the form of flow used in the experimental set-up). No accuracy is quoted below $0.05 \mathrm{~m} / \mathrm{s}$ as linearisation is based on interpolation between 0.05 and $0 \mathrm{~m} / \mathrm{s}$, not on calibration measurements. Hence the reference anemometer meets the mean velocity accuracy requirement of $\pm 0.05 \mathrm{~m} / \mathrm{s}$, in [19].

\subsection{Tracking}

As is evident in Fig. 8 the instantaneous response of the prototype thermal anemometer does not track that of the reference. It is assumed that this is due to the larger thermal mass of the sensor used in the prototype and a consequential slower response time. A comparison of time averaged data from both the prototype and the reference, indicates that the tracking performance of the prototype is more than adequate for this application. This is illustrated in Fig. 9.

Note that a calibration was not undertaken. The data suggests that the apparent discrepancy between the sensor output and the reference could be modelled as a linear relationship, thus including any offset term.

\subsection{Directionality}

Fig. 4 shows that the directional response of the prototype thermal anemometer does not follow that of the reference. It is assumed that this is due to the shielding of off-axis airflow by the prototypes' larger blind spot due to the sensor guard design (shown in Fig. 3).

A more thorough evaluation of the prototype's response to different yaw, pitch, and roll, airflow configurations was possible using the wind tunnel design (all three co-ordinates are individually adjustable), but was not undertaken. Such a study is reserved for a redesigned sensor head with improved directionality performance. Note that directional sensitivity and quality of sensor head guarding, to protect the fragile sensor head in practical use, is a design trade-off.

\subsection{Thermal compensation}

As is evident in Fig. 8 the output of the prototype thermal anemometer is temperature sensitive. This may 
be compensated using King's law or another method. Although some form of self-compensation is desirable, e.g., that used by [29], this option was not available. The determination of the optimal method for thermal compensation is a subject for further study.

\section{Conclusion}

The use of a wind tunnel was necessary to provide the uni-directional horizontal flow test environment necessary for the optimal evaluation of a prototype thermal anemometer.

As shown in Fig. 8, the negative temperature response of the prototype is clearly evident. This disadvantageous aspect requires compensation prior to field use. Similar to the data shown in Fig. 4, but in the controlled (nearisothermal) environment of the experimental apparatus, the linear response of the prototype was evident. This, together with the reduction in price, by one order of magnitude compared to the scientific sensors, shows strong promise for field application subject to the following further developments.

1. A means of compensating the prototype thermal anemometer is necessary to facilitate comparable calculation of drying measure (compared to the reference). The commercial developer was unclear as to whether thermal compensation was most economically performed by hardware or software modifications. It is most important to note that this is essentially an isothermal application since the growth of mushrooms is carried out at a constant temperature [4]. This is a subject for further study.

2. The prototype sensor head guard makes the sensor directionally sensitive. For field use, modification to improve response to airflow on different yaw, pitch or roll, axes is required. Note that the commercial developer is of the opinion that directional sensitivity could be much improved without a significant production cost increase.

\section{Acknowledgements}

We are grateful to Dr Peter Corcoran, UCG and Cora Tine Teo., for the loan of the two prototype anemometers used in this study. Thanks are also due to Mr Oliver Power of the National Electronics Test Centre for the use of the environmental chamber. A final word of thanks are due to Teagasc, the Irish Agriculture and Food Development Authority for their experimental support and funding of this research via a Walsh Fellowship.

\section{References}

[1] G.E. Bowman, Air circulation in mushroom houses, Mushroom Journal 173 (1987) 151-169.

[2] K.M. Lomax, Evaporation power of air, Mushroom Journal 566 (1997) 14-15.

[3] R.L. Edwards, Mushroom-house ventilation: use and misuse, Mushroom Journal 76 (1979) 137-143.

[4] Teagasc, Mushroom growing in bags. Teagasc, Dublin, 1994.

[5] J.J.R. Feddes, J.B. McQuitty, Design of an air-speed sensor system, Canadian Agricultural Engineering 22 (1980) 97-99.

[6] I. Erdebil, J.J. Leonard, Laboratory testing of humidity sensors for use in animal environments, Canadian Agricultural Engineering 34 (1992) 267-271.

[7] H.H. Bruun, Hot-wire Anemometry: Principles and Signal Analysis, Oxford University Press, Oxford, 1995.

[8] C.G. Bowers Jr, D.H. Willits, H.D. Bowen, Comparison of temperature correction methods for hot wire anemometers, ASAE Transactions 31 (1988) 1552-1555.

[9] K. Bremhorst, Effect of fluid temperature on hot-wire anemometers and an improved method of temperature compensation and linearisation without use of small signal sensitivities, Journal of Physics E: Scientific Instrumentation 18 (1) (1985) 43-49.

[10] F.H. Champagne, The temperature sensitivity of hot wires, Proceedings of the '78 dynamic flow conference 1978, pp. 101-114.

[11] M.K. Swaminathan, R. Bacic, G.W. Rankin, K. Sridhar, Improved calibration of hot-wire anemometers, Journal of Physics E: Scientific Instrumentation 16 (1983) 335-338.

[12] H.H. Bruun, M.A. Khan, H.H. Al-Kayiem, A.A. Fardad, Velocity calibration relationships for hot-wire anemometry, Journal of Physics E: Scientific Instrumentation 21 (1988) 225-232.

[13] D.P. Martin, J.V. Ringwood, J.J. Grant, Spatial homogeneity of air in a mushroom tunnel: single vs. boost duct air distribution systems. ASHRAE Transactions 107(1), (2001) 275-284.

[14] P. Freymuth, Hot-wire anemometer thermal calibration errors, Instruments and Control Systems October (1970) 82-83.

[15] A.K. Melikov, G. Langkilde, T.L. Madsen, Impact of velocity and temperature fluctuations on the accuracy of low velocity measurements indoors by thermal anemometers, ASHRAE Transactions 104 (1b) (1998) 1519-1528.

[16] R.D. Fox, R.D. Brazee, A.W. Swank, A tower-mounted calibrator for hot-film anemometers, ASAE Transactions 34 (6) (1991) 2579-2582.

[17] British Standard 1042: Section 2.3 (ISO 7194-1983) Measurement of Fluid Flow in Closed Conduits, BSI, London, 1984.

[18] Z. Popiolek, A.K. Melikov, F.E. Jorgensen, W. Finkelstein, T. Sefker, Impact of natural convection on the accuracy of low velocity measurements by thermal anemometers with omnidirectional sensor, ASHRAE Transactions 104 (1b) (1998) 1507-1518.

[19] Standard 55-1992, Thermal Environmental Conditions for Human Occupancy, ASHRAE, Atlanta, 1992.

[20] A.K. Melikov, Z. Popiolek, W. Finkelstein, T. Sefker, I. Care, Requirements and guidelines for low velocity measurements, ASHRAE Transactions 104 (1b) (1998) 1529-1539.

[21] J.W. Dally, W.F. Riley, K.G. McConnell, Instrumentation for Engineering Measurements, John Wiley, New York, 1993.

[22] US Department of Commerce, Defense, and Transportation, Surface Observations-Federal Meteorological Handbook No. 1, US Government Printing Office, Washington, 1975.

[23] B.W. Olesen, Draught and air velocity measurements. DISA information, no. 24, Technical University of Denmark, 1979, pp. 30-35.

[24] G. Zhang, J.S. Strom, J.C. Bennetsen, Integration time needed for determination of reproducible air velocities in a ventilated room, in: AgEng98 International Conference on Agricultural Engineering, Oslo, vol.1, 1998, pp. 175-176.

[25] R.C. Werner, L.H. Soderholm, R.H. Shaw, Determination of 
acceptable sampling rates for recording wind-speed data, ASAE Transactions 22 (1979) 1175-1185.

[26] J. Grant, Airspeed time average for field use. Personal communication, 1999.

[27] A.E. Perry, Hot-wire Anemometry, Oxford University Press, Oxford, 1982.
[28] L.E. Drain, The Laser Doppler Technique, John Wiley, Chichester, 1980.

[29] H. Stachowiak, S. Lassue, A. Dubernard, E. Gaviot, A thermoelectric sensor for fluid flow measurement: principles, calibration and solution for self temperature compensation, Flow Measurement and Instrumentation 9 (4) (1998) 135-141. 